\title{
Fístula arteriovenosa pós-nefrolitotripsia percutânea
}

\author{
Arteriovenous fistula after percutaneous nephrolithotomy
}

\section{Autores \\ Luis Alberto Batista Peres \\ Sérgio Luiz Bader \\ Rubia Bethania Biela \\ Gabriela Bonissoni \\ Liberali \\ Hospital Universitário da Universidade Estadual do Oeste do Paraná \\ (UNIOESTE)}

Data de submissão: 16/03/2009

Data de aprovação: 13/07/2009

Correspondência para: Luis Alberto Batista Peres

Rua São Paulo, 769/901

Centro, Cascavel/PR

CEP: 85801-020

Tel: (45) 3224-4744

E-mail: peres@certto.com.br

Declaramos a inexistência de conflitos de interesse.

\section{Resumo}

Fístula arteriovenosa (FAV) é uma rara complicação pós-nefrolitotripsia percutânea (NLP). Apresentamos o caso de um paciente de 70 anos, sexo masculino, que apresentou sangramento maciço após NLP, tratado por angioembolização renal superseletiva com implante de stent. Após a embolização, houve resolução do sangramento. FAV é uma complicação incomum da NLP, que pode ser tratada com sucesso com angioembolização.

Palavras-chave: fístula arteriovenosa, nefrolitotomia percutânea, nefrolitotripsia percutânea.

[J Bras Nefrol 2009;31(3):232-234] CElsevier Editora Ltda.

\section{INTRODUÇÃO}

Nefrolitotripsia ultrassônica percutânea, ureterolitotripsia a laser e nefrolitotripsia transureteroscópica são procedimentos em que, por meio de incisões de $1 \mathrm{~cm}$ na pele ou pelas vias urinárias naturais, é possível alcançar os cálculos em qualquer localização. Aparelhos de fibra ótica de fino calibre podem desintegrar cálculos, aplicando energia de várias fontes, como eletro-hidráulica, ultrassônica ou Holmium laser, permitindo um retorno rápido do paciente à sua atividade normal. Esses procedimentos são indicados para cálculos renais duros em razão de sua composição química, ou muito grandes (maiores que $2 \mathrm{~cm}$ ) e que não são resolvidos com litotripsia extracorpórea. ${ }^{1}$

Complicações após a nefrolitotomia têm sido descritas, dentre elas hematúria, fístula artériovenosa (FAV), hematoma perirrenal e laceração arterial. ${ }^{2,3}$ Hematúria severa decorrente de FAV é uma rara

\section{Abstract}

Arteriovenous fistula (AVF) is a rare complication of percutaneous nephrolithotomy (PNL). We report the case of a 70year-old man, who had massive bleeding after PNL and underwent treatment with superselective renal angiographic embolization and stent implantation. Bleeding resolved after embolization. Arteriovenous fistula is an uncommon complication of PNL, which may be successfully treated with angiographic embolization.

Keywords: arteriovenous fistula, percutaneous nephrolithotomy, percutaneous nephrolithotripsy.

complicação de nefrolitotomia percutânea (NLP), que pode ser tratada por angioembolização. ${ }^{4}$

A seguir, apresentamos o relato de um caso raro de FAV após NLP ultrassônica tratada por embolização e implante de stent com sucesso.

\section{Relato Do Caso}

Paciente branco, 70 anos, do sexo masculino, hipertenso de longa data em uso de nifedipina $40 \mathrm{mg} / \mathrm{dia}$ e captopril $100 \mathrm{mg} / \mathrm{dia}$, admitido para abordagem urológica de nefrolitíase bilateral. Ao exame físico: bom estado geral, consciente, lúcido, anictérico, afebril, eupneico, FC = 72 bpm, pressão arterial de $180 / 120 \mathrm{mmHg}$, ausculta cardíaca com hiperfonese de A2 e ausculta pulmonar sem anormalidades, abdome flácido, plano, sem visceromegalias e sem massas palpáveis, extremidades sem edemas. Os exames laboratoriais à admissão revelaram: urina parcial com hematúria microscópica 
e ausência de bactérias, creatinina $1,9 \mathrm{mg} / \mathrm{dL}$, ureia $41,7 \mathrm{mg} / \mathrm{dL}$, hemograma com hemoglobina de $14 \mathrm{~g} / \mathrm{dL}$, hematócrito de $43,8 \%$, leucócitos $10.200 / \mathrm{mm}^{3}$, potássio de $4,20 \mathrm{mEq} / \mathrm{L}$. Urografia excretora realizada previamente ao internamento revelou litíase renal bilateral, rim direito com cálculo em cálice inferior de $1 \mathrm{~cm}$ e cálculo na junção ureteropiélica de $1 \mathrm{~cm}$ e exclusão funcional; e rim esquerdo com cálculo piélico de $2,5 \mathrm{~cm}$ e cálculos caliciais com hidronefrose (Figura 1). Submetido a cistoscopia e cateterismo ureteral direito com mobilização do cálculo para o rim e realizada NLP à direita com punção única de cálice inferior sem incidentes com retirada de dois cálculos, sendo mantida a nefrostomia por 24 horas com alta no segundo dia do pós-operatório sem drenagem pelo orifício da nefrostomia, com levofloxacina $500 \mathrm{mg} / \mathrm{dia}$.

Figura 1. Urografia excretora (radiografia simples e cinco minutos após injeção do contraste) revelou litíase renal bilateral, rim direito com cálculo em cálice inferior de $1 \mathrm{~cm}$ e cálculo na junção ureteropiélica de $1 \mathrm{~cm}$ e exclusão funcional; e rim esquerdo com cálculo piélico de $2,5 \mathrm{~cm}$ e cálculos caliciais com hidronefrose.

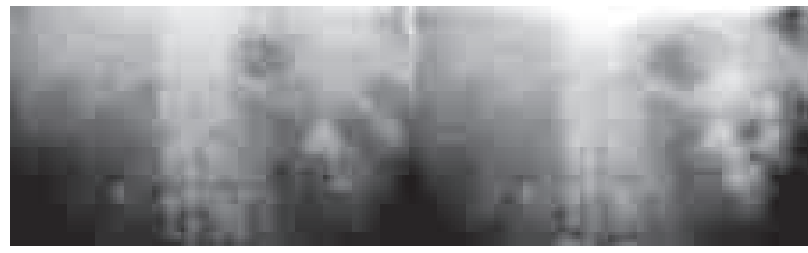

Reinternado sete dias após a alta, o paciente foi submetido à NLP à esquerda e à nefrostomia, com punção única em cálice inferior e fragmentação do cálculo piélico, com retirada de todos os fragmentos. Na evolução, apresentou diurese hematúrica de $5.200 \mathrm{~mL} /$ dia, com retirada da nefrostomia após 48 horas e alta hospitalar com levofloxacina $500 \mathrm{mg} / \mathrm{dia}$. Após 36 horas da alta hospitalar, apresentou quadro neurológico de dificuldade para deambulação e tonturas, sendo reinternado com depleção do volume extracelular. Ao exame neurológico, Glasgow 15 e hemiparesia esquerda. Ressonância magnética revelou artérias vertebrobasilares dilatadas e tortuosas, com deformação da medula oblonga e ponte. Diagnóstico de acidente vascular isquêmico. Medicado com pentoxifilina $400 \mathrm{mg} /$ dia e ácido acetilsalić́lico $100 \mathrm{mg} /$ dia. Apresentou hematúria e volume urinário em torno de $4.000 \mathrm{~mL} /$ dia, sendo mantido com cateterismo vesical e lavagem com solução salina devido à grande formação de coágulos. Houve necessidade de transfusão de duas unidades de concentrado de hemácias. Evoluiu com aumento progressivo da creatinina, com necessidade de diálise peritoneal por 24 horas. Houve remissão do quadro neurológico e persistência da hematúria. Submetido à arteriografia renal bilateral, evidenciou-se FAV à esquerda, com vaso de grande calibre (Figura 2), sendo realizadas embolização e colocação de stent com resolução da fístula AV (Figura 3). Houve remissão da hematúria em 24 horas. Recebeu alta hospitalar após 17 dias de internamento, com função renal normal, sem déficit neurológico e quadro hipertensivo controlado.

Figura 2. Arteriografia renal bilateral evidencia fístula arteriovenosa à esquerda, com vaso de grande calibre.

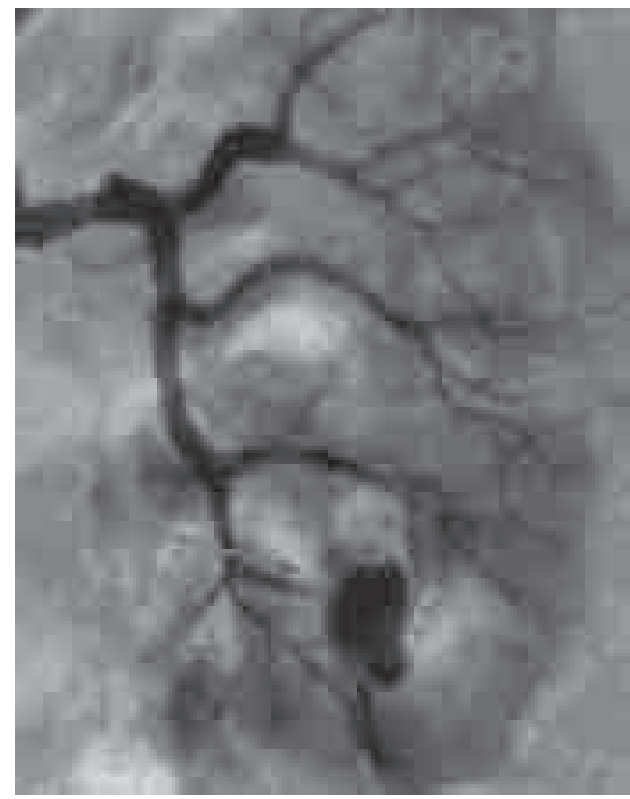

Figura 3. Embolização e colocação de stent com resolução da fístula $A V$ na sequência da arteriografia diagnóstica.

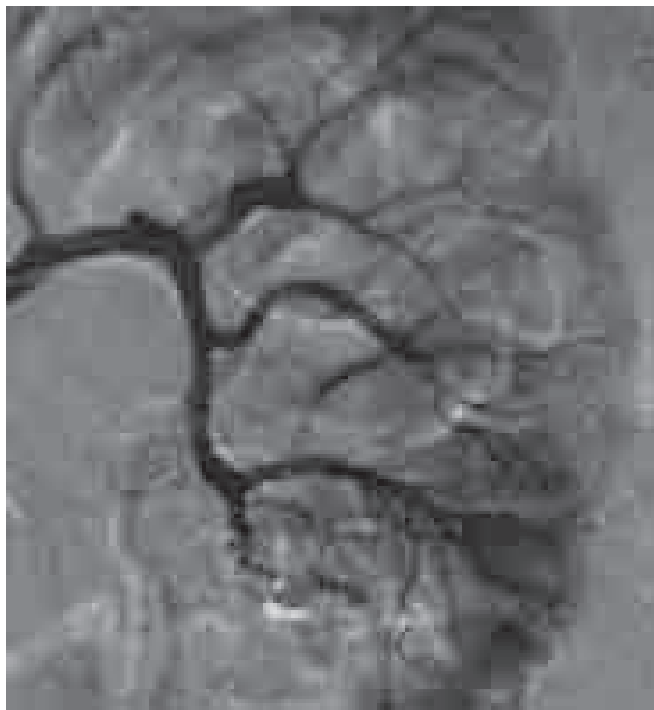

\section{Discussão}

FAV é uma rara complicação de NLP que se apresenta com hematúria severa, cujo tratamento pode ser a angioembolização ${ }^{4}$. Apresentamos o relato de um caso raro de FAV após nefrolitotomia percutânea ultrassônica tratada por embolização e implante de stent com sucesso. 
A NLP ultrassônica é um procedimento utilizado para cálculos renais duros e que não são resolvidos com a litotripsia extracorpórea. Outras modalidades da NLP são a eletro-hidráulica e a Holmium laser. ${ }^{1}$

Algumas complicações após a NLP têm sido descritas, como, por exemplo, hematúria, FAV, hematoma perirrenal e laceração arterial. ${ }^{3,5}$ Hematúria severa decorrente de FAV é uma rara complicação de NLP que pode ser tratada por angioembolização. Em geral, sua incidência se dá nas primeiras três semanas, podendo ocorrer até 13 semanas após o procedimento. ${ }^{4,6}$ Há alguns anos, a embolização superseletiva tem sido considerada o tratamento de escolha para hemorragia maciça após NLP. ${ }^{7}$ Hemorragia severa pode levar à perda do rim., ${ }^{8,9}$

Srivastava et al. ${ }^{3}$ (2005) avaliaram as complicações hemorrágicas da NLP após 1.854 procedimentos e as observaram em 27 (1,4\%) pacientes. Dentre elas, citamse pseudoaneurisma em 13, FAV em seis, combinação de ambos em quatro, laceração de artéria lombar em um e ausência de lesões em três. Em 22 pacientes, a embolização se mostrou bem-sucedida, sendo que em dois a hematúria recorreu. Em análise multivariada, apenas o tamanho do cálculo foi preditor de complicações vasculares.

$\mathrm{O}$ sucesso da angioembolização nesses pacientes tem sido descrito na literatura como superior a $80 \%$, segundo alguns autores..$^{10}$ Há relato de embolização completa da artéria renal com perda do rim. ${ }^{8}$ Além disso, a angioembolização resulta em uma área isquêmica do parênquima renal com efeitos negativos na função do órgão. Poucos estudos têm mostrado os efeitos do procedimento na função renal. ${ }^{11,12,13}$ El-Nahas et al. ${ }^{14}$ (2008) avaliaram efeitos morfofuncionais após três meses em 41 pacientes submetidos ao procedimento de um total de 4.095 que realizaram a NLP, utilizando creatinina sérica, ultrassonografia e cintilografia com DMSA. A creatinina aumentou em três de nove pacientes portadores de rim único, e o seguimento no longo prazo não mostrou comprometimento importante morfofuncional nesses pacientes.

No paciente em questão, chama a atenção o fato de ser paciente idoso com cálculos grandes de difícil abordagem e que necessitou de nefrolitotomia percutânea bilateral em dois tempos. Apresentou na evolução hematúria, injúria renal aguda, provavelmente isquêmica, devido à poliúria pós-desobstrução, acidente vascular cerebral com indicação de antiagregante plaquetário que pode ter agravado a hematúria, com necessidade transfusional. Devido à severidade da hematúria, levantou-se a hipótese de FAV, que foi confirmada por arteriografia renal.

Apresentamos o relato de um caso raro de FAV após NLP ultrassônica tratada por embolização e implante de stent com sucesso.

\section{Conclusões}

NLP é um procedimento seguro para cálculos urinários grandes, sendo raras as complicações, incluindo hematúria. FAV é uma causa de hematúria pósNLP que pode ser tratada por angioembolização. Apresentamos um caso de FAV tratada por angioembolização e implante de stent com sucesso.

\section{REFERÊNCIAS}

1. Yuchico MP, Ko R. The Current Status of Percutaneous Nephrolithotomy in the Management of Kidney Stones. Minerva Urol Nefrol 2008; 60:159-75.

2. El-Nahas AR, Shokeir AA, El-Assmy AM et al. PostPercutaneous Nephrolithotomy Extensive Hemorrhage: a study of risk factors. J Urol 2007; 177:576-9.

3. Srivastava A, Singh KJ, Suri A et al. Vascular Complications after Percutaneous Nephrolithotomy: are there any predictive factors? Urology 2005; 66:38-40.

4. Richstone L, Reggio E, Ost MC et al. Hemorrhage Following Percutaneous Renal Surgery: characterization of angiographic findings. J Endourol 2009; 22:1129-35.

5. El-Nahas AR, Shokeir AA, Mohsen T et al. Functional and Morphological Effects of Postpercutaneous Nephrolithotomy Superselective Renal Angiographic Embolization. J Urol 2007; 71:408-12.

6. Sampaio FJB. Review Anatomy Background for Intrarenal Endourologic Surgery. J Endourol 1992; 6:301-5.

7. Martin X, Murat FJ, Feitosa LC et al. Severe Bleeding after Nephrolithotomy: results of hiperselective embolization. Eur Urol 2000; 37:136-9.

8. Kernohan RM, Johnston LC, Donaldson RA. Bleeding Following Percutaneous Nephrolithotomy Resulting in Loss of the Kidney. Br J Urol 1990; 65:657-8.

9. Patterson DE, Segura JW, Leroy AJ et al. The Etiology and Treatment of Delayed Bleeding Following Percutaneous Lithotripsy. J Urol 1985; 133:447-51.

10. Kessaris DN, Bellmann GC, Paradalidis NP et al. Management of Hemorrhage after Percutaneous Renal Surgery. J Urol 1995; 153:604-8.

11. Poulakis V, Ferakis N, Becht E, Deliveliotis C, Duex M. Treatment of Renal-Vascular Injury by Transcatheter Embolization: immediate and long-term effects on renal function. J Endourol 2006; 20:405-9.

12. Chatziioannou A, Brountzos E, Primetis E et al. Effects of Superselective Embolization for Renal Vascular Injuries on Renal Parenchyma and Function. Eur J Vasc Endovasc Surg 2004; 28:201-6.

13. Perini S, Gordon RL, LaBerge JM et al. Transcatheter Embolization of Biopsy-Related Vascular Injury in the Transplant Kidney: immediate and long-term outcome. J Vasc Interv Radiol 1998; 9:1011-9.

14. El-Nahas AR, Shokeir AA, Mohsen $\mathrm{T}$ et al. Functional and Morphological Effects of Postpercutaneous Nephrolithotomy Superselective Renal Angiographic Embolization. Urology 2008; 71:408-12. 